Working Paper 05-58

Economics Series 27

October 2005
Departamento de Economía Universidad Carlos III de Madrid

Calle Madrid, 126

28903 Getafe (Spain)

Fax (34) 916249875

\title{
A COMPARISON OF THE AVERAGE PREKERNEL AND THE PREKERNEL *
}

\author{
Roberto Serrano ${ }^{1}$ and Ken-Ichi Shimomura ${ }^{2}$
}

\begin{abstract}
We propose positive and normative foundations for the average prekernel of NTU games, and compare them with the existing ones for the prekernel. In our non-cooperative analysis, the average prekernel is approximated by the set of equilibrium payoffs of a game where each player faces the possibility of bargaining at random against any other player. In the cooperative analysis, we characterize the average prekernel as the unique solution that satisfies a set of Nash-like axioms for two-person games, and versions of average consistency and its converse for multilateral settings
\end{abstract}

JEL Classification: C71, C72, C78.

Keywords:. average prekernel, prekernel, Nash set, Nash program, bargaining, consistency, average consistency.

* Acknowledgements: We thank Robert Aumann, Michael Maschler, William Thomson, Federico Valenciano, Oscar Volij and Jose Zarzuelo for useful comments.

Serrano thanks Universidad Carlos III and CEMFI in Madrid for their hospitality, and acknowledges grants from Fundacion Banco Herrero, Universidad Carlos III and the Alfred P. Sloan Foundation. Shimomura acknowledges research support from Japan's Ministry of Education, Culture, Sports, Scienceand Technology, and the Zengin Foundation for Studies on Economics and Finance.

\footnotetext{
${ }^{1}$ Department of Economics, Brown University, Providence, RI 02912, U.S.A.

${ }^{2}$ RIEB, Kobe University, 2-1 Rokkodai, Nada, Kobe 657-8501, Japan
} 


\title{
A Comparison of \\ the Average Prekernel and the Prekernel*
}

\author{
Roberto Serrano ${ }^{\dagger}$ \\ and \\ Ken-Ichi Shimomura ${ }^{\ddagger}$
}

This version: October 2005

\begin{abstract}
We propose positive and normative foundations for the average prekernel of NTU games, and compare them with the existing ones for the prekernel. In our non-cooperative analysis, the average prekernel is approximated by the set of equilibrium payoffs of a game where each player faces the possibility of bargaining at random against any other player. In the cooperative analysis, we characterize the average prekernel as the unique solution that satisfies a set of Nash-like axioms for two-person games, and versions of average consistency and its converse for multilateral settings.

Journal of Economic Literature classification numbers: C71, C72, C78.
\end{abstract}

Keywords: average prekernel, prekernel, Nash set, Nash program, bargaining, consistency, average consistency.

${ }^{*}$ We thank Robert Aumann, Michael Maschler, William Thomson, Federico Valenciano, Oscar Volij and Jose Zarzuelo for useful comments. Serrano thanks Universidad Carlos III and CEMFI in Madrid for their hospitality, and acknowledges grants from Fundacion Banco Herrero, Universidad Carlos III and the Alfred P. Sloan Foundation. Shimomura acknowledges research support from Japan's Ministry of Education, Culture, Sports, Science and Technology, and the Zengin Foundation for Studies on Economics and Finance.

${ }^{\dagger}$ Department of Economics, Brown University, Providence, RI 02912, U.S.A., e-mail: roberto_serrano@brown.edu

${ }^{\ddagger}$ RIEB, Kobe University, 2-1 Rokkodai, Nada, Kobe 657-8501, Japan, e-mail: ken-ichi@rieb.kobe-u.ac.jp 


\section{Introduction}

The prekernel or Nash set of a non-transferable (NTU) coalitional game consists of those payoffs in which each player is in a situation of "bilateral equilibrium" with any other player. ${ }^{1}$ The prekernel was introduced for the class of transferable utility (TU) games in Davis and Maschler (1965) and generalized to the class of NTU games in Serrano (1997). The latter paper, as part of the Nash program for coalitional games (Nash (1950, 1953); see Serrano (2005) for a recent survey), also contained a non-cooperative model of negotiations to support the payoffs in the prekernel. The prekernel was characterized in Peleg (1986) for the class of TU games and in Serrano and Shimomura (1998) for the class of smooth NTU games.

It is important at the outset to clarify our terminology and reconcile it with that found in the literature. While the name prekernel is certainly justified for historical reasons, there are good arguments to also adopt the name Nash set for this solution concept. ${ }^{2}$ Indeed, a payoff $x$ is in the prekernel or Nash set if for every pair of players $i$ and $j$, the point $\left(x_{i}, x_{j}\right)$ is a point of the Pareto frontier of the bilateral problem for $i$ and $j$ where the utility elasticity is unity. That is, $\left(x_{i}, x_{j}\right)$ is a critical point of the Nash product in the bilateral problem's frontier. Thus, it should not come as a surprise that the prekernel or Nash set reduces to a single point in the class of convex bargaining problems and that this point is the Nash solution. Nonetheless, not to bother the reader with the use of both names and following the tradition of coalitional games, we shall use the name prekernel in the rest of this paper.

In multilateral settings, the prekernel is a good description of equilibrium in bilateral bargaining between any pair of players. However, it has an important shortcoming. As pointed out in Moldovanu (1990) and Serrano (1997), it often prescribes the empty set. Following an idea of Maschler and Owen's (1992) for the NTU Shapley value and Dagan and Volij's (1997) for bankruptcy rules, this existence problem has been recently solved in Orshan and Zarzuelo (2000), who propose as an alternative solution concept the average prekernel. ${ }^{3}$ The average prekernel is the

\footnotetext{
${ }^{1}$ See Maschler (1992) for a survey.

${ }^{2}$ Maschler, Owen and Peleg (1988) introduced this solution for the class of bargaining problems.

${ }^{3}$ To continue with our remarks on terminology, we shall use this name to refer to their solution. The one they used, that of bilateral consistent prekernel, is somewhat confusing: first, the average prekernel is not bilaterally consistent; and second, one of the key axioms used in Serrano and Shimomura (1998) to characterize the prekernel
} 
set of efficient payoffs where, for each player, the aggregate (or average) difference of surpluses of a player against all the others is zero. Thus, it always contains the prekernel (because in the latter the difference of surpluses is zero for every pair of players). However, its advantage over the prekernel is that, as Orshan and Zarzuelo (2000) demonstrate, it is non-empty very generally, over a large significant class of NTU games. This result has been refined even further for the intersection of the average prekernel and the core in Orshan, Valenciano and Zarzuelo (2003), who show this intersection is non-empty when the game is "boundary separating."

The purpose of this paper is to clarify the distinction between prekernel and average prekernel, by proposing strategic and axiomatic analyses of the average prekernel. These are to be compared with those for the prekernel, contained in Serrano (1997) and Serrano and Shimomura (1998).

In our non-cooperative analysis, given a status quo payoff, a player is chosen at random every period and asked whether he accepts or rejects the status quo. If he accepts, the status quo is unaltered and a new player will be randomly selected the next period and asked the same question. If he rejects, he will bargain with another player for a redistribution of payoffs, but at the time he has to respond he does not know who will be his opponent in the bilateral bargaining round. In this round, upon rejection of a proposal, the status quo prevails with high probability, while with the rest of probability the rejector has the option of hiring a coalition that includes him but excludes the proposer. Whatever the outcome of this bilateral bargaining round, a new status quo is so determined, followed by the random choice of a new player the next period, and so on. We show that the stationary equilibrium payoffs of this model approximate the average prekernel payoffs as the probability of cooperating with coalitions vanishes. In contrast, the model in Serrano (1997) that yields the prekernel is such that every period a pair of players is chosen at random to bargain if they want to modify the status quo. Thus, while the rules of negotiation in Serrano (1997) lead to a situation of bilateral equilibrium for every pair, a story of "equilibrium in average" is told here.

Likewise, the only differences between the axioms in Serrano and Shimomura (1998) and those used in the present paper are found in the different versions of consistency and its converse. Instead of employing consistency and its converse in the sense of the Davis-Maschler is precisely that of bilateral consistency. 
reduced game, we need average consistency and average converse consistency with respect to certain reduced hyperplane games to characterize the average prekernel over the class of smooth NTU games. ${ }^{4}$ The basis of our characterization is the class of two-player games, where we use the same axioms as in Serrano and Shimomura (1998): non-emptiness, Pareto efficiency, equal treatment for TU games, scale invariance and local independence. As it turns out, one needs to make both changes (consistency versus average consistency, and Davis-Maschler reduced games versus reduced hyperplane games) to go from the axioms of the prekernel to those of the average prekernel. Indeed, we show an impossibility result if one works with average consistency using Davis-Maschler reduced games. Finally, also as the parallel result in Serrano and Shimomura (1998) states, the intersection of the core and the average prekernel is characterized using the same axioms as for the average prekernel, but for the class of smooth games with non-empty cores. In all our characterizations, the axioms utilized are logically independent.

\section{Preliminaries}

Denote by $\mathbb{R}$ the set of the real numbers. If we use an upper case letter to denote a set, its lower case counterpart denotes its cardinality. Thus, let $N$ be a finite set containing at least two elements, and let $n=|N|$. Denote by $\mathbb{R}^{N}$ the set of all functions from $N$ to $\mathbb{R}$. We identify an element $x \in \mathbb{R}^{N}$ with an $n$-dimensional vector whose components are indexed by members of $N$; thus we write $x_{i}$ for $x(i)$. If $x \in \mathbb{R}^{N}$ and $S \subseteq N$, we write $x_{S}$ for the restriction of $x$ to $S$, which is the element of $\mathbb{R}^{S}$ that associates $x_{i}$ with each $i \in S$. We also write $x_{-S}$ to denote $x_{N \backslash S}$ and $x_{-i}$ to denote $x_{N \backslash\{i\}}$.

A player is an element of $N$, and a non-empty subset $S$ of $N$ is a coalition. A payoff to player $i$ is a point of $\mathbb{R}^{\{i\}}$, and a payoff profile for coalition $S$ is a point of $\mathbb{R}^{S}$. The Pareto frontier of a set of payoffs $Y \subseteq \mathbb{R}^{S}$ is:

$$
\partial Y=\left\{y \in Y \mid x_{i}>y_{i} \quad \forall i \in S \quad \text { implies } \quad x \notin Y\right\} .
$$

Definition: The pair $(N, V)$ is a non-transferable utility coalitional game, or simply an (NTU)

\footnotetext{
${ }^{4}$ See Thomson (1995) for a comprehensive survey on consistency. The reader will also find there a useful discussion on consistency and average consistency.
} 
game, if $V$ is a correspondence that associates with every non-empty $S \subseteq N$ a non-empty subset $V(S) \subseteq \mathbb{R}^{S}$ satisfying the following assumptions:

(1) $V(S)$ is closed-valued and comprehensive-valued, i.e., for each $x_{S} \in V(S),\left\{x_{S}\right\}-\mathbb{R}_{+}^{S} \subseteq$ $V(S)$.

(2) For each $x_{S} \in \mathbb{R}^{S}$,

$$
\partial V(S) \cap\left(\left\{x_{S}\right\}+\mathbb{R}_{+}^{S}\right)
$$

and

$$
\partial V(S) \cap\left(\left\{x_{S}\right\}-\mathbb{R}_{+}^{S}\right)
$$

are bounded.

(3) There exists a continuously differentiable representation of $V(N)$, i.e., a continuously differentiable function $g: \mathbb{R}^{N} \rightarrow \mathbb{R}$ such that

$$
V(N)=\left\{x \in \mathbb{R}^{N} \mid g(x) \leq 0\right\}
$$

The interior of $V(N)$ is the set

$$
\operatorname{Int} V(N)=\left\{x \in \mathbb{R}^{N} \mid g(x)<0\right\},
$$

and the Pareto frontier of $V(N)$ is the set $\partial V(N)$ of points $x \in \mathbb{R}^{N}$ such that $g(x)=0$.

(4) $V(N)$ is non-levelled, i.e., for every $x \in \partial V(N)$, the gradient of $g$ at $x$ is positive in all its coordinates, i.e., $\nabla g(x) \gg 0$. We shall write $g_{i}(x)$ for the partial derivative of $g$ at $x \in \partial V(N)$ with respect to component $i \in N$. Thus, $\nabla g(x)=\left(g_{i}(x)\right)_{i \in N}$, so $g_{i}(x)>0$ for all $i \in N$ and for all $x \in \partial V(N)$.

A transferable utility game, or a TU game, is a coalitional game $(N, V)$ defined by a function $v$ that associates with every coalition $S$ a real number $v(S)$ such that

$$
V(S)=\left\{x_{S} \in \mathbb{R}^{S} \mid \sum_{i \in S} x_{i} \leq v(S)\right\}
$$

Abusing notation, we use $(N, v)$ to denote the associated coalitional game. 
A hyperplane game is an NTU game such that the boundary of each $V(S)$ is a hyperplane in $\mathbb{R}^{S}$. A bargaining problem is an NTU game where for all $S \subset N, V(S) \subseteq \Pi_{i \in S} V(\{i\})-\mathbb{R}_{+}^{S}$.

Let $\Gamma$ be a non-empty class of games. A solution on $\Gamma$ is a mapping $\sigma$ which associates with every $(N, V) \in \Gamma$ a (possibly empty) subset $\sigma(N, V)$ of $V(N)$ for every $(N, V) \in \Gamma$.

Let $\Pi^{N}=\{P \subseteq N: p=2\}$, which is the set of two-person coalitions in $N$.

Definition: Let $(N, V)$ be a game, $x \in V(N)$, and $P \in \Pi^{N}$. The two-person (Davis-Maschler) reduced game of $(N, V)$ with respect to $P$ given $x_{-P}$ is the pair $\left(P, V_{x, P}\right)$, consisting of the set $P$ and the correspondence $V_{x, P}$ that associates with every coalition $S \subseteq P$ a subset $V_{x, P}(S)$ of $\mathbb{R}^{P}$, where

$$
V_{x, P}(\{i\})=\left\{y_{i} \in \mathbb{R}^{\{i\}} \mid\left(y_{i}, x_{Q}\right) \in V(\{i\} \cup Q), Q \subseteq N \backslash P\right\}
$$

for each $i \in P$, and

$$
V_{x, P}(P)=\left\{y_{P} \in \mathbb{R}^{P} \mid\left(y_{P}, x_{-P}\right) \in V(N)\right\} .
$$

Thus, given a payoff profile $x$ for the grand coalition $N$, the feasible set for the pair $P$ in the Davis-Maschler reduced game is what remains of $V(N)$ after the players not in $P$ are paid according to $x$. In addition, each player in $P$ expects to be able to cooperate with any of the players not in $P$ provided they are paid their components of $x$. This is the way each player in $P$ finds his "threat utility" against the other player in $P$.

Definition: Let $(N, V)$ be a game, $x \in \partial V(N)$, and $P \in \Pi^{N}$. The two-person reduced hyperplane game of $(N, V)$ with respect to $P$ given $x_{-P}$ is the pair $\left(P, W_{x, P}\right)$, consisting of the set $P$ and the correspondence $W_{x, P}$ that associates with every coalition $S \subseteq P$ a subset $W_{x, P}(S)$ of $\mathbb{R}^{P}$, where

$$
W_{x, P}(\{i\})=V_{x, P}(\{i\})
$$

for each $i \in P$, and

$$
W_{x, P}(P)=\left\{\left(y_{i}, y_{j}\right) \in \mathbb{R}^{P} \mid g_{i}(x)\left(y_{i}-x_{i}\right)+g_{j}(x)\left(y_{j}-x_{j}\right) \leq 0\right\} .
$$

Thus, the reduced hyperplane game has the same feasible sets for the two individuals as the Davis-Maschler reduced game, but it prescribes the hyperplane tangent to the frontier at $x_{P}$ for $P$ : both players make the fictitious assumption that utility is transferable at the rates prescribed by the gradient of the frontier at $x$. Note how reduced hyperplane games are only defined for efficient payoff profiles in the grand coalition. 
The surplus of player $i$ against player $j$ at the payoff vector $x$ is defined as follows:

$$
s_{i, j}(x)=v_{i}\left(x_{-\{i, j\}}\right)-x_{i}
$$

where

$$
v_{i}\left(x_{-\{i, j\}}\right)=\max V_{x,\{i, j\}}(\{i\}) .
$$

That is, the surplus of player $i$ against player $j$ at the payoff profile $x$ is the difference between the highest utility that player $i$ could get without cooperating with $j$ (when paying other players for their resources at the rate prescribed by $x$ ) and the utility that $i$ receives at $x$.

Definition: Let $(N, V)$ be a game. The prekernel of $(N, V)$ is:

$$
\mathcal{P}(N, V)=\left\{x \in \partial V(N) \mid \quad g_{i}(x) s_{i, j}(x)-g_{j}(x) s_{j, i}(x)=0 \quad \forall i, j \in N\right\}
$$

The prekernel of a game is the set of efficient payoff profiles where each player's surplus (when weighted by the corresponding partial derivative of $g$ at $x$ ) against every other player is the same. This is the sense in which each prekernel payoff has the flavor of a "bilateral equilibrium." See Serrano (1997) for an elaboration of this point in a non-cooperative setup, and Peleg (1986) and Serrano and Shimomura (1998) for characterizations of the prekernel. Serrano (1997) also explains how the surplus equations of the prekernel are justified, in spite of the apparent interpersonal utility comparisons.

Definition: Let $(N, V)$ be a game. The average prekernel of $(N, V)$ is:

$$
\mathcal{A P}(N, V)=\left\{x \in \partial V(N) \mid \sum_{j \in N \backslash\{i\}}\left[g_{i}(x) s_{i, j}(x)-g_{j}(x) s_{j, i}(x)\right]=0 \quad \forall i \in N\right\} .
$$

The average prekernel was introduced in Orshan and Zarzuelo (2000). It consists of those efficient payoff profiles in which each player is in a bilateral equilibrium situation only in average: it is possible that player $i$ 's surplus against player $j$ exceeds that of player $j$ against $i$, but this is offset by exactly the opposite situation with the players other than $j$.

\section{Strategic Bargaining and the Average Prekernel}

In this section we introduce a modification of the non-cooperative model proposed in Serrano (1997). The result will be a strategic bargaining model that approximates the payoff profiles in the average prekernel as stationary equilibrium payoffs. 
For this section, we shall need the following additional assumptions on the game $(N, V)$ :

(5) $V(N)$ is a convex set.

(6) $V(S) \subseteq\left\{x_{S} \in \mathbb{R}^{S} \mid\left(x_{S}, 0_{T \backslash S}\right) \in V(T)\right\}$ whenever $S \subseteq T$.

Convexity of $V(N)$ will be needed to ensure feasibility of the outcomes in the non-cooperative procedure: as we shall describe shortly, many of them are lotteries on the set $V(N)$. As for monotonicity, it will be used to construct equilibria in which it is always optimal for the proposer to form the grand coalition.

Finally, for each player $i \in N$ and each payoff profile $x_{-i} \in \mathbb{R}^{N \backslash\{i\}}$, we write $g^{i}: \mathbb{R}^{N \backslash\{i\}} \rightarrow$ $\mathbb{R}$ to denote the $i$-th coordinate Pareto projection $g^{i}\left(x_{-i}\right)$, i.e., $\left(g^{i}\left(x_{-i}, x_{-i}\right) \in \partial V(N)\right.$. Our assumptions on the game ensure that this concept is well defined.

Description of the non-cooperative model. Time runs discretely from $-\infty$ to $+\infty$. In period $t$, let $x_{t}$ denote the status quo payoff vector determined by play in period $t-1$. There is a fixed protocol of players, say, $1,2, \ldots, n$. In period $t$ one of the players, say $i \in N$, is asked whether he accepts the status quo $x_{t}$ or he rejects it. If he accepts it, play goes to period $t+1$, when player $i+1(\bmod n)$ will be asked the same question, and $x_{t+1}=x_{t}$. If player $i$ rejects the status quo, he makes a proposal $y_{t}$ to the other players, who must all simultaneously respond to it. If $y_{t}$ is accepted, it becomes the new status quo: $x_{t+1}=y_{t}$. If at least one of the players in $N \backslash\{i\}$ rejects player $i$ 's proposal, each of the players $j \in N \backslash\{i\}$ is selected at random with probability $1 /(n-1)$ to bargain bilaterally with player $i$.

The bargaining procedure between players $i$ and $j$ in period $t$ is as follows. Let player $j$ be the one selected to bargain with player $i$ in period $t$. Denote the status quo in period $t$ simply by $x$.

1. The proposer or offerer is chosen at random with probability $1 / 2$. Denote the proposer by $o=i, j$ and the responder by $r=i, j, r \neq o$. The proposer $o$ makes a feasible offer $\left(z_{o}, z_{r}\right)$, i.e., $\left(z_{o}, z_{r}, x_{-\{i, j\}}\right) \in V(N)$.

2. The responder $r$ may accept or reject the offer. 
- If $r$ accepts it, play goes to period $t+1$, where

$$
x_{t+1}=\left(z_{o}, z_{r}, x_{-\{i, j\}}\right) .
$$

- If $r$ rejects the offer,

- With probability $1-\rho>0$, play goes to period $t+1$, where

$$
x_{t+1}=\left(v_{r}\left(x_{-\{i, j\}}\right), g^{o}\left(v_{r}\left(x_{-\{i, j\}}\right), x_{-\{i, j\}}\right), x_{-\{i, j\}}\right) .
$$

- With probability $\rho$, play goes to period $t+1$ with $x_{t+1}=x_{t}$.

A word on the interpretation of what happens following a rejection is called for. With probability $1-\rho$, the responder executes his threat against the proposer: he gets to utilize the resources of a coalition that includes him but excludes the proposer and pays the members of that coalition their components of $x$. The proposer's payoff is determined by whatever is left of $V(N)$ after taking into account this contract between the responder and the outside coalition (note how the grand coalition always forms; other coalition structures are not our focus in this paper). With probability $\rho$, the status quo prevails.

As in Serrano (1997), one should assume that either players are myopic and care only about present payoffs, or that there are $n$ large populations (each being a "social class") underlying the model, and in each period one representative of some of these "social classes" is given the opportunity of modifying the social status quo.

To analyze this non-cooperative model, we shall use the following solution concept:

Definition: An equilibrium of the non-cooperative model is a strategy profile satisfying the following requirements:

(i) Given a sequence of status quo vectors $\left\{x_{t}\right\}_{t}$, each player $i \in N$ plays according to stationary subgame perfect equilibrium strategies in each period $t$, when deciding the acceptance of $x_{t}$ and in the subgames consisting of bilateral bargaining.

Steady state: the sequence of status quo vectors exhibits cycles of length $n$, i.e., $x_{t}=x_{t+n}$ for all $t$. 
The equilibrium and the sequence of status quo vectors will in general depend on $\rho$. Ultimately, what we shall be interested in are sequences with the property that $\lim _{\rho \rightarrow 1} x_{t}=x$ for all $t$. This will be referred to as converging sequences.

First, it is easy to see that existence of an equilibrium obtains. Assume that the individually rational region of $V(N)$, denoted $V(N)_{+}$, is non-empty. Then, thanks to the other assumptions on the coalitional game, one can concentrate on $V(N)_{+}$as the set of payoffs where all action takes place. Consider the mapping $\phi_{i}: V(N)_{+} \mapsto V(N)_{+}, \phi_{i}(x)=y$, which, given the status quo $x$, is constructed by player $i$ offering to each player $j$ the expected payoff $y_{j}$ that $j$ would obtain from rejecting $i$ 's proposal. That is,

$$
\begin{gathered}
y_{j}=\frac{1}{n-1}\left[\frac{1}{2}(1-\rho) v_{j}\left(x_{-\{i, j\}}\right)+\frac{1}{2} \rho x_{j}+\frac{1}{2} g^{j}\left((1-\rho) v_{i}\left(x_{-\{i, j\}}\right)+\rho x_{i}\right)\right]+\frac{n-2}{n-1} x_{j}, \quad \forall j \neq i ; \\
y_{i}=g^{i}\left(y_{-i}\right) .
\end{gathered}
$$

This mapping is continuous. Now consider the composition mapping $\phi_{1}\left(\phi_{2}\left(\ldots \phi_{n}(\cdot)\right)\right)$. This also goes from $V(N)_{+}$into itself, and being a composition of continuous mappings, it is also continuous. Therefore, by Brouwer's fixed point theorem, it has a fixed point. This establishes existence of an equilibrium in our sense, i.e., a sequence of status quo vectors whose cycle lasts $n$ periods.

We can now state and prove the main result of this section:

Theorem 1 Let $(N, V)$ be an NTU game satisfying Assumptions (1)-(6). Then, if $z$ is the limit as $\rho \rightarrow 1$ of a converging sequence of equilibrium payoff profiles of the non-cooperative model, $z \in \mathcal{A P}(N, V)$.

Proof : Consider a sequence of equilibria of the non-cooperative model, which for each $\rho<1$, yields a payoff profile $x(\rho)$. For the moment, fix $\rho<1$, but to economize on notation, write $x$ instead of $x(\rho)$.

Player $i$, asked in period $t$ about the status quo, must consider whether bargaining or not against a randomly chosen opponent. For the status quo $x$ to remain unchanged, the following 
conditions are required:

$$
x_{i} \geq \sum_{j \in N \backslash\{i\}} \frac{1}{n-1}\left[\frac{1}{2}\left((1-\rho) v_{i}\left(x_{-\{i, j\}}\right)+\rho x_{i}\right)+\frac{1}{2} g^{i}\left((1-\rho) v_{j}\left(x_{-\{i, j\}}\right)+\rho x_{j}, x_{-\{i, j\}}\right)\right] \quad \forall i \in N .
$$

The right hand side is the expected payoff that player $i$ can get by forcing a rejection (for instance, by offering zero to some players). Note how, by Assumption (6), in the bilateral bargaining subgames a proposer always wishes to get an acceptance from the responder by leaving him exactly indifferent to his continuation payoff. This explains the last term of the right hand side.

It is easy to see that, as $\rho \rightarrow 1$, the limit of the sequence of equilibrium payoff profiles $x(\rho)$ must be efficient: to see this, examine the right hand side of the above inequalities and note that, if $\lim _{\rho \rightarrow 1} x(\rho)$ is inefficient, we have that $\lim _{\rho \rightarrow 1} g^{i}\left(x_{-i}(\rho)\right)>\lim _{\rho \rightarrow 1} x(\rho)$, contradicting the inequalities of the system when $\rho$ is close enough to 1 . Therefore, in the limit the above system is one of $n$ equalities.

Continuing to write $x$ instead of the more explicit $x(\rho)$ in the above inequalities, we can rewrite them as follows:

$$
x_{i} \geq \sum_{j \in N \backslash\{i\}} \frac{1}{n-1}\left[\frac{1}{2} v_{i}\left(x_{-\{i, j\}}\right)+\frac{1}{2} \frac{g^{i}\left((1-\rho) v_{j}\left(x_{-\{i, j\}}\right)+\rho x_{j}, x_{-\{i, j\}}\right)-\rho x_{i}}{1-\rho}\right] \quad \forall i \in N .
$$

Next we take limits as $\rho \rightarrow 1$, whose existence follows from the assumptions made on the game. Further, from the argument in the previous paragraph, in the limit we can replace the inequality with an equality. Using L'Hopital's rule and denoting $\lim _{\rho \rightarrow 1} x(\rho)=z$, we have that:

$$
z_{i}=\sum_{j \in N \backslash\{i\}} \frac{1}{n-1}\left[\frac{1}{2} v_{i}\left(z_{-\{i, j\}}\right)+\frac{1}{2}\left(z_{i}-\frac{g_{j}(z)}{g_{i}(z)}\left(z_{j}-v_{j}\left(z_{-\{i, j\}}\right)\right)\right)\right] \quad \forall i \in N,
$$

which can be easily transformed into the equations of the average prekernel. Therefore, $z \in$ $\mathcal{A P}(N, V)$.

We note that we do not have conditions to guarantee that all equilibrium sequences converge. The result only says that, when they do, the corresponding payoff is in the average prekernel. Also, we do not know whether for each payoff profile in the average prekernel one can find a corresponding converging equilibrium sequence. Therefore, our procedure is only indicative 
of the kind of bargaining behind this solution concept, and it is far from delivering its full implementation, an interesting question for further research.

The most substantive difference between this non-cooperative model of bargaining and that found in Serrano (1997) is that each player faces the random possibility of bargaining against any player to affect the status quo. In contrast, in the model of Serrano (1997), the status quo may be modified by the bargaining between a specific pair of players $\{i, j\}$. This brings out quite clearly the distinction between the average prekernel and the prekernel from a strategic stand-point. We turn now to axiomatics.

\section{Axiomatic Analysis}

In this section we characterize the average prekernel by means of seven logically independent axioms. The results here are to be compared with the closely related results in Serrano and Shimomura (1998). A comparison with the axiomatic result in Orshan and Zarzuelo (2000) will also be provided.

Let $\Gamma$ be a non-empty class of games, and $\sigma$ a solution on $\Gamma$. Then, we define the following properties.

Non-emptiness: For each $(N, V) \in \Gamma, \sigma(N, V) \neq \emptyset$.

Pareto efficiency: For each $(N, V) \in \Gamma, \sigma(N, V) \subseteq \partial V(N)$.

Let $(N, v)$ be a TU game, and $i, j$ be two distinct players in $N$. Then $i$ and $j$ are substitutes in $(N, v)$ if $v(S \cup\{i\})=v(S \cup\{j\})$ for all $S \subseteq N \backslash\{i, j\}$.

Equal treatment for TU games: If $(N, v) \in \Gamma$ is a TU game and $i$ and $j$ are substitutes in $(N, v)$, $x \in \sigma(N, v)$ implies that $x_{i}=x_{j}$.

Let $(N, V)$ be a game, $\alpha \in \mathbb{R}_{++}^{N}$, and $\beta \in \mathbb{R}^{N}$. For each coalition $S$, we define the function $\lambda_{S}^{\alpha, \beta}$ from $\mathbb{R}^{S}$ to itself by

$$
\lambda_{S}^{\alpha, \beta}\left(x_{S}\right)=\left(\alpha_{i} x_{i}+\beta_{i}\right)_{i \in S}
$$

for each $x_{S} \in \mathbb{R}^{S}$. We then define $\lambda^{\alpha, \beta}(V)$ as the correspondence that associates with every coalition $S$ a set

$$
\lambda^{\alpha, \beta}(V)(S)=\left\{y \in \mathbb{R}^{N}: \text { there exists } \quad x_{S} \in V(S) \mid y_{S}=\lambda_{S}^{\alpha, \beta}\left(x_{S}\right)\right\}
$$


That is, these two definitions simply describe positive affine transformations of the utility scales. Scale invariance: For each $(N, V) \in \Gamma$, for each $\alpha \in \mathbb{R}_{++}^{N}$ and each $\beta \in \mathbb{R}^{N}, \sigma\left(N, \lambda^{\alpha, \beta}(V)\right)=$ $\lambda_{N}^{\alpha, \beta}(\sigma(N, V))$.

Local independence: For each $(N, V),\left(N, V^{\prime}\right) \in \Gamma$ and $x \in \sigma(N, V)$, if

$x \in \partial V(N) \cap \partial V^{\prime}(N)$,

$V(S)=V^{\prime}(S)$ for each $S \neq N$ and

$\nabla g(x)$ is proportional to $\nabla g^{\prime}(x)$,

where $g$ and $g^{\prime}$ are representations for $V(N)$ and $V^{\prime}(N)$, respectively, then $x \in \sigma\left(N, V^{\prime}\right)$.

For a discussion of each of these axioms, see Serrano and Shimomura (1998).

Proposition 1 Let $\Gamma^{\{i, j\}}$ be the class of two-person games $(\{i, j\}, V)$ satisfying Assumptions (1)-(4). Then a solution on $\Gamma^{\{i, j\}}$ satisfies non-emptiness, Pareto efficiency, equal treatment for TU games, scale invariance and local independence if and only if it is $\mathcal{A} \mathcal{P}$.

Proof : The proof is identical to that of Proposition 1 in Serrano and Shimomura (1998) after one observes that, over the considered class of games, $\mathcal{A} \mathcal{P}=\mathcal{P}$.

Thus, over the class of two-player games, the average prekernel coincides with the prekernel. If the set $V(\{i, j\})$ is convex and we have a bargaining problem, the average prekernel consists of a unique payoff profile, the one prescribed by the Nash solution.

Next, we shall present the axioms that will be operative in multilateral settings. To facilitate the comparison with the prekernel, we also state the axioms used in Serrano and Shimomura (1998).

Bilateral consistency: For each $(N, V) \in \Gamma$ and each $x \in \sigma(N, V),\left(P, V_{x, P}\right) \in \Gamma$ and $x_{P} \in$ $\sigma\left(P, V_{x, P}\right)$ for each $P \in \Pi^{N}$.

Bilateral consistency says that the solution should be invariant to projections to two-player games, provided players have the expectations embodied in the bilateral Davis-Maschler reduced 
game..$^{5}$

Converse consistency: For each $(N, V) \in \Gamma$ and each $x \in \partial V(N)$, if $\left(P, V_{x, P}\right) \in \Gamma$ and $x_{P} \in$ $\sigma\left(P, V_{x, P}\right)$ for each $P \in \Pi^{N}$, then $x \in \sigma(N, V)$.

For a solution satisfying converse consistency, in order to impose its recommendations on a society, an arbitrator should simply make sure that the restrictions of the recommended payoff vector to each pair of players agrees with the solution (when the feasible possibilities for the players in the pair are described by the relevant reduced game).

Serrano and Shimomura (1998) show that, over the class of smooth games, the prekernel $\mathcal{P}$ is the only solution satisfying bilateral consistency, converse consistency, and the five axioms for two-player games used in Proposition $1 .^{6}$

We next formulate concepts of average consistency. Average bilateral consistency: For each $(N, V) \in \Gamma$ and each $x \in \sigma(N, V),\left(P, V_{x, P}\right) \in \Gamma$ for each $P \in \Pi^{N}$, and $x=\sum_{P \in \Pi^{N}} \frac{2}{n(n-1)}\left(y_{P}, x_{-P}\right)$ for some $y_{P} \in \sigma\left(P, V_{x, P}\right)$.

This is weaker than bilateral consistency: given a payoff profile $x$ in the solution, it is required only that $x$ be the average of $n(n-1) / 2$ vectors: each of these, fixing the payoffs $x_{-P}$, consists of solution payoffs in each bilateral reduced game $\left(P, V_{x, P}\right)$. Note how single-valuedness of the solution in reduced games is not required.

Average converse consistency: For each $(N, V) \in \Gamma$, if $\left(P, V_{x, P}\right) \in \Gamma$ for each $P \in \Pi^{N}$ and $x=\sum_{P \in \Pi^{N}} \frac{2}{n(n-1)}\left(y_{P}, x_{-P}\right)$ for some $y_{P} \in \sigma\left(P, V_{x, P}\right)$, then $x \in \sigma(N, V)$.

Contrary to the relationship between bilateral consistency and its average counterpart, average converse consistency is stronger than converse consistency. First, it is not required that $x$ be efficient. In addition, the requirement for the decentralization of $x$ is now much weaker: we require from a payoff $x$ that, if $x$ is the average of solution/projection payoffs over all the associated bilateral reduced games, $x$ must be recommended to the whole society as a solution point.

A class of games is rich if it includes games of more than two players and it contains all

\footnotetext{
${ }^{5} \mathrm{~A}$ strengthening of this property is consistency. A solution $\sigma$ on a class $\Gamma$ satisfies consistency if the same condition as above holds, but for all subsets $P$, that is, not only restricted to those subsets $P$ of $N$ of cardinality 2 (see Thomson (1995)).

${ }^{6}$ The results in Serrano and Shimomura (1998) are robust to a stronger version of converse consistency that replaces " $x \in \partial V(N)$ " with " $x \in V(N)$." This observation is of interest for the sequel.
} 
two-person games.

In cooperative game theory, Pareto efficiency is usually required as a desirable axiom. We study now the implications of Pareto efficiency, the other four axioms in Proposition 1 for twoplayer games, and average bilateral consistency and average converse consistency in our next impossibility result. 
Theorem 2 Let $\Gamma_{0}$ be a rich class of games satisfying Assumptions (1)-(4) that includes games $(N, V)$ in which $V(N)$ is a convex set whose Pareto frontier does not contain flat segments. There is no solution on $\Gamma_{0}$ satisfying Pareto efficiency, average bilateral consistency and average converse consistency, as well as the following four axioms for two player games: non-emptiness, equal treatment for TU games, scale invariance and local independence.

Proof : For $n=2$, average bilateral consistency reduces to bilateral consistency and average converse consistency to converse consistency. Therefore, by Proposition 1, the only solution satisfying the seven axioms considered is $\mathcal{P}=\mathcal{A P}$ over this class.

Let $n \geq 3$ and consider an NTU game $(N, V)$ in which $V(N)$ is a convex set such that $\partial V(N)$ does not contain flat segments. ${ }^{7}$ Suppose a solution $\sigma$ satisfies all seven axioms. Let $x \in \sigma(N, V)$. By Pareto efficiency and average bilateral consistency of $\sigma$, and by our choice of the game excluding flat segments in $\partial V(N)$, we have that $\sigma$ must satisfy bilateral consistency. That is, for all $P \in \Pi^{N}, x_{P} \in \sigma\left(P, V_{x, P}\right)$. Since for all two-player games, we know that $\sigma$ coincides with the prekernel, we have that for all $P \in \Pi^{N}, x_{P} \in \mathcal{P}\left(P, V_{x, P}\right)$. By the converse consistency of the prekernel, we get that $x \in \mathcal{P}(N, V)$. Therefore, for every game $(N, V)$ where $V(N)$ is a convex set for which $\partial V(N)$ does not include flat segments, $\sigma(N, V) \subseteq \mathcal{P}(N, V)$.

Now consider $x \in \mathcal{P}(N, V)$. By the bilateral consistency of the prekernel, we know that for all $P \in \Pi^{N}, x_{P} \in \mathcal{P}\left(P, V_{x, P}\right)$, and given that $\sigma=\mathcal{P}$ for two-player games, we get that for all $P \in \Pi^{N}, x_{P} \in \sigma\left(P, V_{x, P}\right)$. Therefore, by the average converse consistency of $\sigma, x \in \sigma(N, V)$. Hence, $\mathcal{P}(N, V) \subseteq \sigma(N, V)$.

Thus, over the class of games $(N, V)$ where $V(N)$ is a convex set for which $\partial V(N)$ does not include flat segments, the only possibility is that $\sigma$ is the prekernel. However, as shown by the following example, $\mathcal{P}$ does not satisfy average converse consistency on a game in this class: let $N=\{1,2,3\}, V(\{i\})$ is the non-positive real half-line for each $i \in N$, and $V(N)$ is the set whose Pareto frontier is a curved concave surface passing through the points $z^{1}=(1 / 3,11 / 36,13 / 36)$, $z^{2}=(13 / 36,1 / 3,11 / 36)$ and $z^{3}=(11 / 36,13 / 36,1 / 3)$. We impose also that at each of these $z^{h}$,

\footnotetext{
${ }^{7}$ The next steps in this proof and the bulk of the proof of Theorem 3 are familiar in theorems that use consistency. They comprise what Thomson (1995) calls the "elevator lemma."
} 
for the two players $i$ and $j$ who do not receive a payoff of $1 / 3, g_{i}\left(z^{h}\right)=g_{j}\left(z^{h}\right)$. As for the twoplayer coalitions, denote by cch the comprehensive hull of the convex hull of the given set. (To fit this example within all our assumptions on NTU games, the reader can consider a smooth and strictly comprehensive version of the frontier of the two-player coalitions outside of the relevant range. This will make no difference.) Then:

$$
\begin{aligned}
& V(\{1,2\})=\left\{\left(x_{1}, x_{2}\right):\left(x_{1}, x_{2}\right) \in \operatorname{cch}\{(1,0),(0,2 / 3)\}\right\} \\
& V(\{1,3\})=\left\{\left(x_{1}, x_{3}\right):\left(x_{1}, x_{3}\right) \in \operatorname{cch}\{(2 / 3,0),(0,1)\}\right\} \\
& V(\{2,3\})=\left\{\left(x_{2}, x_{3}\right):\left(x_{2}, x_{3}\right) \in \operatorname{cch}\{(1,0),(0,2 / 3)\}\right\}
\end{aligned}
$$

Let $x=(1 / 3,1 / 3,1 / 3)$. Note how, by local independence, $(11 / 36,13 / 36) \in \mathcal{P}\left(\{1,2\}, V_{x,\{1,2\}}\right)$, $(13 / 36,11 / 36) \in \mathcal{P}\left(\{1,3\}, V_{x,\{1,3\}}\right)$, and $(11 / 36,13 / 36) \in \mathcal{P}\left(\{2,3\}, V_{x,\{2,3\}}\right)$. Therefore, the vector $x=(1 / 3,1 / 3,1 / 3)$, which is obtained as the average of $z^{1}, z^{2}$ and $z^{3}$, ought to be in $\mathcal{P}(N, V)$ if the prekernel satisfied average converse consistency. However, $x \notin \mathcal{P}(N, V)$.

Remark 1: An impossibility result can be also obtained on any rich class of games by using all the same axioms and requiring local independence for $n$-player games. To see this, pick $x \in \sigma(N, V)$. By efficiency, $x \in \partial V(N)$. By local independence, if necessary, construct a game $(N, U)$ with a strictly concave frontier and such that $x \in \sigma(N, U)$. After this change, the steps of the proof are identical as those in the proof of Theorem 2.

Remark 2: The proof of Theorem 2 shows that the prekernel does not satisfy average converse consistency. For completeness, let us note that the average prekernel does not satisfy it either (as the same example shows). In addition, while the prekernel satisfies bilateral consistency, and hence its average counterpart, the average prekernel does not even satisfy average bilateral consistency. To see this, just use the same example as in the last step of the proof of Theorem 2, and construct a symmetric Pareto frontier of $V(N)$. Then, the point $x=(\lambda, \lambda, \lambda) \in \partial V(N)$ will be in the average prekernel. However, this point cannot be obtained as an average over two-person coalitions $P$ of points $\left(y_{P}, \lambda\right)$ satisfying that $y_{P} \in \mathcal{A P}\left(P, V_{x, P}\right)$.

As implied by one of the steps in the proof of Theorem 2, efficiency and average consistency imply consistency when one considers NTU games. This fact and considerations similar to those 
in Remark 2 suggest the following alternative definitions.

Average bilateral consistency with respect to reduced hyperplane games: For each $(N, V) \in \Gamma$ and each $x \in \sigma(N, V),\left(P, W_{x, P}\right) \in \Gamma$ for each $P \in \Pi^{N}$, and $x=\sum_{P \in \Pi^{N}} \frac{2}{n(n-1)}\left(y_{P}, x_{-P}\right)$ for some $y_{P} \in \sigma\left(P, W_{x, P}\right)$.

That is, much in the spirit of Shapley's $\lambda$-transfer principle, using as utility weights the gradient of $\partial V(N)$ at $x$, we can make the thought experiment that utility is transferable at those rates. Then, this version of average bilateral consistency has the same interpretation as the previous one: each payoff in the solution is the expectation of solution points to bilateral games where the threat points are determined by the Davis-Maschler logic and the feasible set is the half-space generated by the utility weights.

Average converse consistency with respect to reduced hyperplane games: For each $(N, V) \in \Gamma$, if $\left(P, W_{x, P}\right) \in \Gamma$ for each $P \in \Pi^{N}$ and $x=\sum_{P \in \Pi^{N}} \frac{2}{n(n-1)}\left(y_{P}, x_{-P}\right)$ for some $y_{P} \in \sigma\left(P, W_{x, P}\right)$, then $x \in \sigma(N, V)$.

Remark 3: On a rich class of games, $\mathcal{A P}$ satisfies average bilateral consistency with respect to reduced hyperplane games and average converse consistency with respect to reduced hyperplane games.

Our main result in this section follows:

Theorem 3 Let $\Gamma_{0}$ be a rich class of games satisfying Assumptions (1)-(4). A solution on $\Gamma_{0}$ satisfies average bilateral consistency with respect to reduced hyperplane games, average converse consistency with respect to reduced hyperplane games, and the following five axioms for twoplayer games (non-emptiness, Pareto efficiency, equal treatment for TU games, scale invariance and local independence) if and only if it is $\mathcal{A P}$.

Proof : By Proposition 1 and Remark 3, the solution $\mathcal{A P}$ on $\Gamma_{0}$ satisfies the seven axioms listed. Now we prove uniqueness.

Let $(N, V) \in \Gamma_{0}$, and let $\sigma$ be a solution on $\Gamma_{0}$ that also satisfies the seven axioms of the Theorem. We prove that $\sigma(N, V)=\mathcal{A P}(N, V)$. The proof for $n=1$ is trivial. We have already proven the case of $n=2$ (Proposition 1 and Remark 3). Then consider the case of $n \geq 3$. 
Let $x \in \mathcal{A} \mathcal{P}(N, V)$. By the average bilateral consistency with respect to reduced hyperplane games of $\mathcal{A P}$, there exist $n(n-1) / 2$ vectors $\left(y_{P}, x_{-P}\right), y_{P} \in \mathcal{A P}\left(P, W_{x, P}\right)$ for all $P \in \Pi^{N}$ such that $x=\sum_{P \in \Pi^{N}} \frac{2}{n(n-1)}\left(y_{P}, x_{-P}\right)$. But for every two-player game $\left(P, W_{x, P}\right)$, if $\sigma$ satisfies all seven axioms, $\sigma\left(P, W_{x, P}\right)=\mathcal{A P}\left(P, W_{x, P}\right)$. Hence, there exist $n(n-1) / 2$ vectors $\left(y_{P}, x_{-P}\right)$, $y_{P} \in \sigma\left(P, W_{x, P}\right)$ for all $P \in \Pi^{N}$ such that $x=\sum_{P \in \Pi^{N}} \frac{2}{n(n-1)}\left(y_{P}, x_{-P}\right)$. By the average converse consistency with respect to reduced hyperplane games of $\sigma, x \in \sigma(N, V)$. Hence, $\mathcal{A P}(N, V) \subseteq$ $\sigma(N, V)$.

To prove the opposite inclusion, we follow analogous steps. Take $x \in \sigma(N, V)$. By average bilateral consistency with respect to reduced hyperplane games of $\sigma$, for each $P \in \Pi^{N},\left(P, W_{x, P}\right) \in$ $\Gamma_{0}$ and $x$ can be obtained as the average of vectors $y_{P}, x_{-P}$ for some $y_{P} \in \sigma\left(P, W_{x, P}\right)$. Since over the considered class of two-player games, $\sigma=\mathcal{A P}$, we have that for each $P \in \Pi^{N}, y_{P} \in$ $\mathcal{A P}\left(P, W_{x, P}\right)$, and by the average converse consistency with respect to reduced hyperplane games of the average prekernel, $x \in \mathcal{A P}(N, V)$. This establishes that $\sigma(N, V) \subseteq \mathcal{A} \mathcal{P}(N, V)$. Therefore, $\sigma(N, V)=\mathcal{A P}(N, V)$.

Remark 4: The basis of the characterization in Theorem 3 is to pin down the solution on twoperson games with the five axioms imposed there, and to extend it to multilateral settings with the versions of average consistency and its converse for reduced hyperplane games. This was also the methodology in Serrano and Shimomura (1998), except that there bilateral consistency and its converse were used, instead of their average versions for reduced hyperplane games. In contrast, the axiomatic result in Orshan and Zarzuelo (2000) is based on characterizing the solution first on the class of hyperplane games. They then require their versions of consistency and its converse only over this class and use local independence for the extension of the result to general smooth NTU games. Note, for example, how their version of consistency (2-CO) is violated by $\mathcal{A P}$ outside of the class of hyperplane games because it is not generally single-valued. Remark 5: Theorem 3 can also be stated over classes of games that are not rich. For example, the characterization would extend over the class of games where feasible sets are convex.

Next we show that the seven axioms used in the characterization are logically independent. In each example, the axiom in brackets is the one violated by the solution proposed. 
Example 4.1 [non-emptiness]: For every $(N, V) \in \Gamma_{0}$, let $\sigma(N, V)=\emptyset$. Then $\sigma$ vacuously satisfies all the conditions of Theorem 3 except non-emptiness for two-person games.

Example 4.2 [Pareto efficiency]: For every two-person game $(P, V)$, define $b(P, V)=\left(v_{i}\right)_{i \in P}$ if $\left(v_{i}\right)_{i \in P} \in \operatorname{Int} V(P)$ and $b(P, V)=\mathcal{A P}(P, V)$ otherwise. For every $(N, V) \in \Gamma_{0}$, let

$$
\sigma(N, V)=\left\{x \in V(N) \mid x=\sum_{P \in \Pi^{N}} \frac{2}{n(n-1)}\left(y_{P}, x_{-P}\right) \quad \text { for some } \quad y_{P} \in b\left(P, W_{x, P}\right)\right\} .
$$

Then $\sigma$ violates Pareto efficiency for two-person games whenever $\left(v_{i}\right)_{i \in P}$ is in the interior of $V(P)$. Clearly, it satisfies non-emptiness, equal treatment for TU games, scale invariance and local independence over two-person games. By construction, $\sigma$ also satisfies average bilateral consistency with respect to reduced hyperplane games and average converse consistency with respect to reduced hyperplane games.

Example 4.3 [equal treatment for TU games]: For every $(N, V) \in \Gamma_{0}$, let

$$
\sigma(N, V)=\partial V(N)
$$

Then $\sigma$ satisfies all the conditions of Theorem 3 except equal treatment for two-person TU games. That $\sigma$ satisfies all the other axioms for two-person games is obvious. In addition, $\sigma$ satisfies bilateral consistency with respect to reduced hyperplane games, and hence, also its weaker average version. Finally, it also satisfies average converse consistency with respect to reduced hyperplane games because these reduced games are defined only for efficient payoff profiles.

Example 4.4 [scale invariance]: For every $(N, V) \in \Gamma_{0}$, let

$$
\sigma(N, V)=\left\{x \in \partial V(N) \mid \sum_{j \in N \backslash\{i\}}\left[s_{i, j}(x)-s_{j, i}(x)\right]=0 \quad \forall i \in N\right\} .
$$

This is a version of the average prekernel, except that the surpluses are not weighted by the value of the partial derivatives of $g$ at the Pareto frontier of $V(N)$. Then it is easy to see that $\sigma$ violates scale invariance for two-person games, while it clearly satisfies all the other axioms for two-person games. Since the solution is based on a certain average surplus for each player, it satisfies the two average consistency axioms with respect to reduced hyperplane games.

Example 4.5 [local independence]: For every two-person game $(P, V)$, define $a(P, V)=$ $\left(a_{i}(P, V)\right)_{i \in P}$ by

$$
a_{i}(P, V)=\max \left\{x_{i} \in \mathbb{R}^{\{i\}} \mid\left(x_{i}, v_{j}\left(x_{-P}\right)\right) \in V(P), \quad P=\{i, j\}\right\} .
$$


For every $(N, V) \in \Gamma_{0}$, let

$$
\sigma(N, V)=\left\{x \in \partial V(N) \mid \quad x=\sum_{P \in \Pi^{N}} \frac{2}{n(n-1)}\left(y_{P}, x_{-P}\right)\right\}
$$

for each $P=\{i, j\}$ and for some

$$
\left.y_{P}=\left(y_{i}, y_{j}\right) \in \partial W_{x,\{i, j\}}(\{i, j\}) \cap\left[\left(v_{k}\left(x_{-\{i, j\}}\right)\right)_{k=i, j}, a\left(\{i, j\}, W_{x,\{i, j\}}\right)\right]\right\},
$$

where $[c, d]=\{(1-t) c+t d \mid 0 \leq t \leq 1\}$ for each $c, d \in \mathbb{R}^{\{i, j\}}$. That is, for every player $i \in N$, $x_{i}$ can be expressed as the average of maximal points of the feasible set $W_{x,\{i, j\}}(\{i, j\})$ on the segment connecting $\left(v_{k}\left(x_{-\{i, j\}}\right)\right)_{k=i, j}$ to $a\left(\{i, j\}, W_{x,\{i, j\}}\right)$ if $x \in \sigma(N, V)$. Note that $\sigma$ is a sort of average of Kalai- Smorodinsky bargaining solutions. Thus, it satisfies all the conditions of Theorem 3 for two-person games except local independence. By construction, it also satisfies the two average consistency axioms with respect to reduced hyperplane games.

Example 4.6 [average bilateral consistency with respect to reduced hyperplane games]: Let $(N, V) \in \Gamma_{0}$. Let $\sigma(N, V)=\mathcal{A} \mathcal{P}(N, V)$ when $n=1,2$, and $\sigma(N, V)=\partial V(N)$ for $n \geq 3$. By Proposition1, $\sigma$ satisfies the five axioms imposed on two-person games. Further, it is easy to see that average converse consistency with respect to reduced hyperplane games is also satisfied (see the argument in Example 4.3). To see that $\sigma$ viollates average bilateral consistency with respect to reduced hyperplane games, consider the 3-player TU game in which $v(N)=1$ and $v(S)=0$ for all other $S$, and take $x=(0.7,0.2,0.1) \in \sigma(N, v)$. However, $x$ is not the average of the three vectors $\left(y_{P}, x_{-P}\right)$ for $y_{P} \in \sigma\left(P, W_{x, P}\right)$ for each two-player reduced game, i.e., $(0.45,0.45,0.1)$, $(0.4,0.2,0.4)$ and $(0.7,0.15,0.15)$.

Example 4.7 [average converse consistency with respect to reduced hyperplane games]: For every $(N, V) \in \Gamma_{0}$, let $\sigma(N, V)=\mathcal{P}(N, V)$. By Proposition 1, $\sigma$ satisfies all the axioms for two-player games. Moreover, $\mathcal{P}$ satisfies bilateral consistency, and therefore, average bilateral consistency (with respect to Davis-Maschler reduced games and with respect to reduced hyperplane games). To see that $\mathcal{P}$ violates average converse consistency with respect to reduced hyperplane games, consider the same game at the end of the proof of Theorem 2, but where $\partial V(N)$ is the hyperplane of equation $x_{1}+x_{2}+x_{3}=1$. Then, $x=(1 / 3,1 / 3,1 / 3)$ is the average of the three vectors $z^{h}=\left(y_{P}, x_{-P}\right)$ with $y_{P} \in \mathcal{P}\left(P, W_{x, P}\right)$ for each $P \in \Pi^{N}$. However, $x \notin \mathcal{P}(N, V)$. 
As in Serrano and Shimomura (1998), we next investigate the implications of the same sets of seven axioms on the class of games with non-empty cores.

Definition: Let $(N, V)$ be a game, $S$ a non-empty subset of $N$, and $x \in \mathbb{R}^{N}$. Then we say that $S$ can improve upon $x$ if there is $y \in V(S)$ such that $y_{i}>x_{i}$ for all $i \in S$. The core of $(N, V)$ is:

$$
\mathcal{C}(N, V)=\{x \in V(N) \mid \text { There is no coalition that can improve upon } x\} .
$$

Definition: Let $(N, V)$ be a game. The intersection of the core and the prekernel of $(N, V)$ is:

$$
\mathcal{P}^{*}(N, V)=\mathcal{C}(N, V) \cap \mathcal{P}(N, V) .
$$

Definition: Let $(N, V)$ be a game. The intersection of the core and the average prekernel of $(N, V)$ is:

$$
\mathcal{A P}^{*}(N, V)=\mathcal{C}(N, V) \cap \mathcal{A P}(N, V)
$$

We can show the following in exactly the same way as for Proposition1 .

Proposition 2 Let $\Gamma_{c}^{\{i, j\}}$ be the class of two-person games $(\{i, j\}, V)$ satisfying Assumptions (1)-(4) and with non-empty cores. Then a solution on $\Gamma_{c}^{\{i, j\}}$ satisfies non-emptiness, Pareto efficiency, equal treatment for TU games, scale invariance and local independence if and only if it is $\mathcal{A} \mathcal{P}^{*}$.

The proof simply follows from the fact that for two-player games $\mathcal{P}^{*}=\mathcal{A P}^{*}$. Serrano and Shimomura (1998) show that, over a rich class of smooth $n$-player games, $\mathcal{P}^{*}$ is the only solution satisfying bilateral consistency, converse consistency, and the group of five axioms for two-player games in Proposition 2. Similarly, using the following remark, we can prove the theorems below as we have done for Theorems 2 and 3 .

Remark 6: On a class of games satisfying Assumptions (1)-(4) with non-empty cores containing all the two-person games with the same properties, $\mathcal{A P}^{*}$ satisfies average bilateral consistency with respect to reduced hyperplane games and average converse consistency with respect to reduced hyperplane games, because so do $\mathcal{A P}$ and $\mathcal{C}$. 
Theorem 4 Let $\Gamma_{c}$ be the class of games satisfying Assumptions (1)-(4) with non-empty cores containing all the two-person games with the same properties and also containing games $(N, V)$ in which $V(N)$ is a convex set whose Pareto frontier does not contain flat segments. There is no solution on $\Gamma_{c}$ satisfying Pareto efficiency, average bilateral consistency and average converse consistency, as well as the following four axioms for two-player games: non-emptiness, equal treatment for TU games, scale invariance and local independence.

Theorem 5 Let $\Gamma_{c}$ be the class of games satisfying Assumptions (1)-(4) with non-empty cores containing all the two-person games with the same properties. A solution on $\Gamma_{c}$ satisfies average bilateral consistency with respect to reduced hyperplane games, average converse consistency with respect to reduced hyperplane games, and the following five axioms for two-player games (non-emptiness, Pareto efficiency, equal treatment for TU games, scale invariance and local independence) if and only if it is $\mathcal{A P}^{*}$.

It can be shown by modifying the seven examples that followed the proof of Theorem 3 that the same seven axioms are also logically independent over the smaller class of games $\Gamma_{c}$.

\section{Conclusion}

We have obtained positive and normative foundations of the average prekernel, and we have compared them to those for the prekernel. The prekernel captures naturally those payoff profiles where players find themselves in a situation of "bilateral equilibrium." Given its frequent existence difficulties, it is convenient to settle for the weaker property of "bilateral equilibrium in average" that the average prekernel describes. Both the strategic and axiomatic analyses performed here

provide support to this notion. It will be interesting to test the differences between the two solution concepts in specific applications. 


\section{References}

Dagan, N. and O. Volij (1997), "Bilateral Comparisons and Consistent Fair Division Rules in the Context of Bankruptcy Problems," International Journal of Game Theory 26, 11-26.

Davis, M. and M. Maschler (1965), "The Kernel of a Cooperative Game," Naval Research Logistics Quarterly 12, 223-259.

Maschler, M. (1992), "The Bargaining Set, Kernel and Nucleolus," in Handbook of Game Theory with Economic Applications (vol. I), R. J. Aumann and S. Hart (eds.), North Holland, New York, 591-667.

Maschler, M. and G. Owen (1992), "The Consistent Shapley Value for Games without Side Payments," in R. Selten (ed.) Rational Interactions: Essays in Honor of J. C. Harsanyi, Springer Verlag, Berlin, 5-12.

Maschler, M., G. Owen and B. Peleg (1988), "Path Leading to the Nash Set," in The Shapley Value; Essays in Honor of L. S. Shapley, A. Roth (ed.), Cambridge University Press, Cambridge, 321-330.

Moldovanu, B. (1990), "Stable Bargained Equilibria for Assignment Games without Side Payments," International Journal of Game Theory 19, 171-190.

Nash, J. F. (1950), "The Bargaining Problem," Econometrica 18, 155-162.

Nash, J. F. (1953), "Two Person Cooperative Games," Econometrica 21, 128-140.

Orshan, G. and J. Zarzuelo (2000), "The Bilateral Consistent Prekernel for NTU Games," Games and Economic Behavior 32, 67-84.

Orshan, G., F. Valenciano and J. Zarzuelo (2003), "The Bilateral Consistent Prekernel, the Core and NTU Bankruptcy Problems," Mathematics of Operations Research 28, 268-282.

Peleg, B. (1986), "On the Reduced Game Property and its Converse," International Journal of Game Theory 15, 187-200. 
Serrano, R. (1997), "Reinterpreting the Kernel," Journal of Economic Theory 77, 58-80.

Serrano, R. (2005), "Fifty Years of the Nash Program, 1953-2003," Investigaciones Economicas 29, 219-258.

Serrano, R. and K.-I. Shimomura (1998), "Beyond Nash Bargaining Theory: the Nash Set," Journal of Economic Theory 83, 286-307.

Thomson, W. (1995), "Consistent Allocation Rules," Working Paper, University of Rochester. 